\title{
La importancia de la convivencia armónica en el desarrollo humano integral de los estudiantes
}

\author{
Mary Lorena Maldonado De Janón \\ marymaldonado27@hotmail.com \\ p7001256586@ucvvirtual.edu.pe \\ Universidad César Vallejo \\ Piura - Perú
}

\section{RESUMEN}

El presente trabajo de investigación busca mejorar la convivencia armónica de los estudiantes del octavo grado a través del fortaleciendo de su desarrollo humano integral. Para conseguirlo, se propuso un programa de convivencia armónica para contribuir al enriquecimiento de los conocimientos básicos en valores, aplicación y mejora del comportamiento y lazos familiares. Para esto, se determinaron las actividades a desarrollarse conjuntamente con charlas, talleres, apoyo psicológico. Todo esto ha beneficiado positivamente a las relaciones interpersonales, comportamiento, trabajo en equipo dentro del salón de clases y fuera del mismo. Este estudio posee un enfoque cuantitativo, de tipo aplicativo, correlacional, transversal debido a su alcance temporal y diseño no experimental. Como instrumentos se aplicó un cuestionario donde se incluyó las dos variables y la observación a una muestra de 30 estudiantes, que es el total de la población considerada. Posteriormente se aplicó el análisis descriptivo, estadístico; donde se evidenció que el desarrollo humano integral incide sobre la convivencia armónica ya que existe una correlación de $0,681^{* *}$, considerada muy alta en el nivel 0,01 bilateral entre las variables estudiadas, bajo la aplicación de la prueba no paramétrica Rho de Spearman. Es decir, a medida que una variable aumente la otra también lo hará.

Palabras Clave: comportamiento; convivencia armónica; desarrollo humano integral; valores. 


\title{
The importance of harmonious coexistence in the integral human development of students
}

\begin{abstract}
This study seeks to improve the harmonious coexistence of eighth-grade students by strengthening their integral human development. A harmonious coexistence program was proposed to enrich basic knowledge in values, application, and the improvement of behavior and family ties. For this, the activities to develop together with talks, workshops, and psychological support were determined. All this has positively benefited interpersonal relationships, behavior, teamwork within the classroom and outside of it. This study has a quantitative, applicative, correlational, cross-sectional approach due to its temporal scope and a non-experimental design. The instruments were an observation sheet and questionnaire applied in a sample of 30 students, which is the total population considered. Subsequently, descriptive and statistical analysis was applied for the interpretation of the collected data. It was evident that integral human development affects harmonious coexistence since there is a correlation of $0.681 * *$, considered very high at the bilateral level 0.01 among the variables studied, under applying the non-parametric Spearman Rho test. In this sense, we could interpret that as one variable increases, the other will also increase.
\end{abstract}

Keywords: behavior; harmonious coexistence; integral human development; values.

Artículo recibido: 20. Julio. 2021

Aceptado para publicación: 18. Agosto. 2021 Correspondencia: marymaldonado27@ hotmail.com

Conflictos de Interés: Ninguna que declarar 


\section{INTRODUCCIÓN}

A lo largo del tiempo, los valores y la práctica de los mismos han quedado rezagados, transformando a las actuales generaciones y limitándoles en el correcto actuar. Como consecuencia, podemos evidenciar que un gran número de niños, niñas, jóvenes y adolescentes se han distanciado de sus raíces conocidas como costumbres y tradiciones. Todos estos cambios paulatinos y otros abruptos, han generado el desapego de las buenas acciones, modales, comportamiento, los cuales perjudican a la sociedad.

Gracias a los diferentes acuerdos y tratados a nivel internacional, se está tratando consolidar una educación en valores, una educación que les permita a los individuos crecer y desarrollarse en el marco de los buenos actos, decisiones, conducta. Pero pese a los intentos, con el paso de los años evidenciamos actos y decisiones equívocas por parte de nuestros gobernantes. Sin duda alguna, es una lucha constante el tratar de establecer un ejemplo correcto a seguir. No obstante, existen seres ejemplares capaces de proyectar logros y cambios positivos a lo largo de la historia.

Si nos remontamos al siglo $\mathrm{V}$ a. C. y partimos del pensamiento de los primeros sofistas de la argumentación lógica; ellos se orientaron al relativismo moral y fueron considerados como relativistas subjetivos ya que rechazaron toda ley moral universal. Establecieron que cada persona posee su verdad moral y emplean su capacidad para determinar lo bueno o malo por encima de la verdad. Posteriormente, los pensamientos de Sócrates (470-399 a.C.), Platón (427-347 a.C.) y Aristóteles (348-322 a.C.) dieron paso a un nuevo pensar, donde las malas acciones están ligadas a la ignorancia del ser. Si se conoce el bien, se debe actuar conforma a él y el valor es su virtud. Por lo tanto, su objetivo esencial es vivir feliz y racionalmente. Estos pensamientos, claramente se oponen a los primeros filósofos ya que parten del análisis de los errores para descubrir la verdad mediantes preguntas y respuestas.

Con base en el estudio Aristotélico de Aaros (2003) podemos establecer que la palabra ética es el resultado de la unión de êthos (carácter) y éthos (hábitos, costumbres), donde se puede concluir que la formación del carácter es a partir de la costumbre, más no es el resultado del destino o azar. Consecuentemente, esto ha llevado al surgimiento de la moral (derivada del latín moralis), lo cual hace referencia a las costumbres.

Si avanzamos en la historia, la filosofía medieval o religiosa hasta el siglo VIII establece que la razón y la fe se complementan, por tanto amar a Dios es ley moral y la 
interiorización es el camino. Santo Tomás de Aquino (1224-1274) nos presenta la declaración de pruebas referentes a la existencia de Dios, donde el hombre es desplazado y Dios es la superioridad ya que reconoce y respeta su libertad. Con lo cual, el individuo debe vivir por su inteligencia y voluntad respetando el orden natural.

En cuanto a filosofía moderna del sigo XVI-XVIII, surgen nuevos filósofos y consigo temas predominantes y nuevas leyes para la libertad. Entre ellos, se destaca Immanuel Kant (1724-1804) quien deduce que el individuo debe respetar a los demás y a sí mismo, donde la sinceridad es exigida y posteriormente, contradice a Tomás de Aquino enfocándose en la parte racional del ser. Siguiendo esta línea, Jeremy Bethan (17481834) señaló que todo acto realizado por el hombre debe ser juzgado.

Avanzando en la línea de tiempo, en la filosofía contemporánea del siglo XIX-XX ocurrieron cambios sociales, políticos, culturales y surgió el utilitarismo donde lo bueno es lo útil. Feuerbach (1804-1872), rechaza totalmente la idea sobre la existencia de Dios y alude que es una invención del individuo sufrido. Karl Marx (1818-1883) reconoce que el hombre requiere de acciones prácticas, por ende elimina toda base de ética e incluye una nueva moral como justificación para referirse a eventos económicos. Él junto a Engels (1820-1895) dentro de sus planteamientos, establecen nuevos caminos de libertad, justicia y felicidad.

En este sentido, Nietzsche (1844-1900) define que la naturaleza del hombre es la irracionalidad y para alcanzar su plenitud debe vivir más allá de la nominación del mal y del bien. Distingue dos tipos de hombre y por consiguiente dos clases de moral, la primera se refiere a los esclavos con la moral para ser sometidos y la segunda se refiere a los hombres con moral superior, quienes demuestran hostilidad, confianza en sí mismo, orgullo propio y burla hacia sus inferiores. Rechaza contundentemente los pensamientos de los filósofos griegos Sócrates, Platón, Aristóteles y enfatiza que entender la vida desde la razón es una total equivocación. Además, critica la moral que se comprende por la timidez, miedo, vergüenza y la moral racional.

Dando paso a la filosofía existencialista del siglo XIX-XX, la cual surgió como resultado de las diversas opiniones, pensamientos y reacciones de las teorías predominantes. Podemos resaltar el pensamiento de Kaufmann (1975), quien lo describe como la no aceptación a pertenecer a una escuela de pensamiento y al rechazo 
debido a la insatisfacción de creer en una filosofía tradicional, superficial y alejada de la verdadera realidad.

La presente investigación se realizó en una institución educativa privada de Ecuador, en la cual ciertos estudiantes del octavo grado manifestaban un cierto nivel de comportamiento errático, una mezcla de lenguaje soez y actitudes agresivas ante una discusión. Generalmente, al culminar el 7mo grado, padres de familia o representantes legales buscan nuevas instituciones para que sus hijos/as prosigan con sus estudios, debido al tipo de institución o al cambio que desean optar. Esto contribuye de manera positiva a que las instituciones no solo fiscales, sino también particulares tengan una gran diversidad en sus instituciones, específicamente en el 8vo grado. Pero, ciertamente no saben qué valores comparten sus alumnos, cuál es su comportamiento ante las diferentes situaciones, si sabe expresarse correctamente ante una discusión. Parte del proceso de admisión de las diferentes entidades educativas es evaluar el conocimiento adquirido de los grados de educación anteriores, test psicológico y dependiendo de éstos son aceptados o rechazados. En cambio, otras instituciones educativas, no creen que estas evaluaciones sean adecuadas para continuar con sus estudios y mucho menos para las edades en las que se encuentran.

En el transcurso del periodo lectivo, se evidencia la riqueza académica y valoral de cada uno de ellos, por lo que suele ser difícil para docentes y autoridades lidiar con ciertos actos indisciplinarios o en el peor de los casos con agresiones físicas.

Martínez et ál. (2018) reconoce que le valor es el núcleo de la distinción moral por la que cada persona opta, donde la vivencia de nivel particular, grupal u singular da paso a la existencia y reconocimiento de un valor.

Bandura (1977) en su teoría del aprendizaje social hace referencia a tres modelos con base en el comportamiento, el primero un modelo vivo que se basa en el individuo real quien manifiesta su comportamiento. El segundo de instrucción verbal, donde se enlaza las explicaciones, descripciones y el tercero simbólico, donde se denota la influencia de personajes reales o ficticios. El tercero es el simbólico en el cual el individuo recibe y/o acepta la influencia de otros, sean estos reales o ficticios.

En su teoría cognitiva, Bruner (1961) establece que el individuo desde temprana edad contribuye a la solución de conflictos de manera activa e inteligente, con características, habilidades, destrezas semejantes al adulto. Vygotsky (1978) desde la perspectiva del 
enfoque constructivista nos indica que el conocimiento es un proceso de interacción entre el sujeto y el medio, contando lo cultural, social y físico.

Los dos psicólogos antes mencionados vieron en el docente a un facilitador del conocimiento, siendo éste el responsable de edificar estructuras mentales y aprendizajes complejos.

En la Teoría de la zona de desarrollo próximo, Vygotsky (1931) nos recuerda la importancia del desarrollo integral dentro del crecimiento de las personas, estableciendo un tiempo real como resultado de los logros realizados en el instante y el potencial que son los objetivos que las personas pueden lograr de manera independiente.

Piaget (1963) referente a la Teoría del desarrollo moral, deduce que con el paso del tiempo el individuo desarrolla una moral más compleja a medida que aumente su capacidad cognitiva. En este sentido, Kohlberg (1984) desarrolló ciertas reglas y normas, las cuales son parte del pensamiento de la persona referente a la moralidad, donde la toma de decisiones le ha conllevado a reconocer sus acciones malas y buenas.

\section{ESTRATEGIAS METODOLÓGICAS O MATERIALES Y MÉTODOS}

Considerando el objeto de estudio y su enfoque, se consideró una metodología cuantitativa, como lo indica Cienfuegos y Cienfuegos (2016) se aplican operaciones aritméticas las cuales son parte de la dicotomía experimental dentro del experimento. De tipo aplicativo, Murillo (2008) nos indica que es práctica porque se busca la correcta aplicación de todos los conocimientos adquiridos y la adquisición de nuevos, con lo que posteriormente se sintetizará e implementará en la práctica.

Díaz y Calzadilla (2016), consideran que el tipo correlacional busca descifrar si existe o no alguna relación, profundizando en el grado y la forma entre las propiedades de los objetivos.

Su corte es transversal como Sampieri (2014) lo establece, debido a su único momento para la recolección de datos y su diseño es no experimental ya que se ha llevado a cabo sin ninguna manipulación de las variables.

En la presente investigación se evidenció dos variables, como variable independiente convivencia armónica y como variable dependiente desarrollo humano integral, apoyadas de sus correspondientes dimensiones. Se elaboró un cuestionario como instrumento para la recolección de información por cada una de las variables. Este 
cuestionario fue validado por cinco expertos con el máximo título en el ámbito educativo. Para constatar la confiabilidad del mismo, se aplicó el alfa de Cronbach.

En este sentido, la recolección de información se la realizó en un solo momento, los estudiantes llenaron el cuestionario bajo la perspectiva vivida durante su 5to, 6to y $7 \mathrm{mo}$ año de educación general básica en la/las escuelas que han cursado.

Los datos obtenidos se los llevó a una hoja de cálculo especificando sus variables y dimensiones, los cuales pasaron por procesos estadísticos correspondientes y el software SPSS, versión 25. De igual manera, se aplicó la Rho de Spearman para comprobar la hipótesis y la aplicación de procesos estadígrafos que han permitido generar tablas de frecuencia relativa y absoluta.

\section{RESULTADOS Y DISCUSIÓN}

El presente trabajo contó con una prueba de normalidad de Shapiro - Wilk entre las dos variables, ya que la población es de 30 estudiantes.

\section{Tabla 1.}

\section{Prueba de normalidad.}

\begin{tabular}{l|c|c|c} 
& \multicolumn{3}{|c}{ Shapiro-Wilk } \\
& Estadístico & gl & Sig. \\
\hline Convivencia armónica &, 773 & 30 &, 000 \\
\hline Desarrollo humano integral &, 942 & 30 &, 102 \\
\hline
\end{tabular}

Nota: Elaborado por: Mary Lorena Maldonado De Janón.

En la Tabla 1, podemos observar que la primera puntuación no es normal ya que $\mathrm{p}<0,05$, por esta razón se justifica la utilización de la estadística no paramétrica. En la segunda puntuación se evidencia que es débil.

En este sentido, se procedió a aplicar la prueba no paramétrica de Rho de Spearman entre las dos variables. 
Tabla 2.

Correlación no paramétrica.

\begin{tabular}{|c|c|c|c|c|}
\hline & & & $\begin{array}{c}\text { Convivencia } \\
\text { armónica }\end{array}$ & $\begin{array}{c}\text { Desarrollo } \\
\text { humano integral }\end{array}$ \\
\hline \multirow{6}{*}{$\begin{array}{l}\text { Rho de } \\
\text { Spearman }\end{array}$} & \multirow{3}{*}{$\begin{array}{l}\text { Convivencia } \\
\text { armónica }\end{array}$} & $\begin{array}{l}\text { Coeficiente de } \\
\text { correlación }\end{array}$ & 1,000 &, $681^{* *}$ \\
\hline & & Sig. (bilateral) & . &, 000 \\
\hline & & $\mathbf{N}$ & 30 & 30 \\
\hline & \multirow{3}{*}{$\begin{array}{l}\text { Desarrollo } \\
\text { humano } \\
\text { integral }\end{array}$} & $\begin{array}{l}\text { Coeficiente de } \\
\text { correlación }\end{array}$ &, $681^{* *}$ & 1,000 \\
\hline & & Sig. (bilateral) & ,000 & . \\
\hline & & $\mathbf{N}$ & 30 & 30 \\
\hline
\end{tabular}

**. La correlación es significativa en el nivel 0,01 (bilateral).

Nota: Elaborado por: Mary Lorena Maldonado De Janón.

En la tabla 2, de acuerdo al baremo de estimación referente a la correlación de Spearman, se puede observar que existe una correlación positiva fuerte. Por lo que se pudo concluir que la primera variable tiene una significativa relación con la segunda.

Procesados los datos obtenidos y aplicando los procesos mencionados anteriormente, se obtuvo:

Tabla 3.

Resultado de las dimensiones de la Variable Independiente Convivencia Armónica.

\begin{tabular}{lcccccc}
\hline INTERVALO & $\mathbf{f}$ & $\mathbf{\%}$ & $\mathbf{f}$ & $\boldsymbol{\%}$ & $\mathbf{f}$ & $\boldsymbol{\%}$ \\
\hline Alto & 22 & $74 \%$ & 25 & $82 \%$ & 4 & $50 \%$ \\
Medio & 7 & $22 \%$ & 6 & $18 \%$ & 15 & $27 \%$ \\
Bajo & 1 & $3 \%$ & 0 & $0 \%$ & 11 & $23 \%$ \\
\hline TOTAL & $\mathbf{3 0}$ & $\mathbf{1 0 0 \%}$ & $\mathbf{3 0}$ & $\mathbf{1 0 0 \%}$ & $\mathbf{3 0}$ & $\mathbf{1 0 0 \%}$ \\
\hline
\end{tabular}

Nota. Datos tomados del Cuestionario aplicado. Elaborado por: Mary Lorena Maldonado De Janón.

En la Tabla 3 se evidencian las dimensiones de la primera variable, las cuales corresponden a inclusividad, trabajo en equipo y diversidad. 


\section{Tabla 4.}

Resultados de las dimensiones de la variable dependiente: Desarrollo Humano Integral.

\begin{tabular}{lcccccccc}
\hline INTERVALO & $\mathbf{f}$ & $\boldsymbol{\%}$ & $\mathbf{f}$ & $\boldsymbol{\%}$ & $\mathbf{f}$ & $\boldsymbol{\%}$ & $\mathbf{f}$ & $\boldsymbol{\%}$ \\
\hline Alto & 18 & $60 \%$ & 21 & $71 \%$ & 16 & $53 \%$ & 17 & $57 \%$ \\
Medio & 12 & $40 \%$ & 8 & $29 \%$ & 14 & $47 \%$ & 12 & $41 \%$ \\
Bajo & 0 & $0 \%$ & 0 & $0 \%$ & 0 & $0 \%$ & 1 & $2 \%$ \\
\hline \multicolumn{1}{c}{ TOTAL } & $\mathbf{3 0}$ & $\mathbf{1 0 0 \%}$ & $\mathbf{3 0}$ & $\mathbf{1 0 0 \%}$ & $\mathbf{3 0}$ & $\mathbf{1 0 0 \%}$ & $\mathbf{3 0}$ & $\mathbf{1 0 0 \%}$ \\
\hline
\end{tabular}

Nota. Datos tomados del Cuestionario aplicado. Elaborado por: Mary Lorena Maldonado De Janón.

Con base en la Tabla 4 podemos observar las dimensiones ética, moral y cívica, espiritualidad o interioridad, cognitiva y afectiva respectivamente.

Tabla 5.

Tablas cruzadas: Convivencia armónica * Desarrollo humano integral. Convivencia armónica* Desarrollo humano tabulación cruzada

Recuento

\begin{tabular}{llcc}
\hline & & $\begin{array}{c}\text { Desarrollo humano integral } \\
\text { AL TO }\end{array}$ & Total \\
\hline \multirow{2}{*}{ Convivencia } & ALTO & 64 & 64 \\
armónica & MEDIO & 32 & 32 \\
& BAJO & 5 & 5 \\
\hline Total & & $\mathbf{1 0 0}$ & $\mathbf{1 0 0}$ \\
\hline
\end{tabular}

Nota. Datos tomados del Cuestionario aplicado. Elaborado por: Mary Lorena Maldonado De Janón.

En la Tabla 5, evidenciamos que el 64\% de los estudiantes encuestados se encuentra en el nivel alto, un $31 \%$ de dichos estudiantes en un nivel medio y el $5 \%$ en el nivel bajo respectivamente.

\section{Discusión}

De acuerdo con el objetivo general planteado en la presente investigación se puede concluir que es muy evidente la relación existente entre las variables. Miño (2018) 
manifiesta que la convivencia armónica es esencial para quien busca una calidad educativa, ya que está fuertemente ligada al bienestar que se puede percibir dentro del salón de clases. Ciertamente, Barrios (2016) basado en su percepción del enfoque constructivista sociocultural, sugiere que el estudiante divisa como lugar idóneo la escuela, donde puede desarrollar y resolver su desarrollo integral, dilemas interpersonales, valores, entre otros.

Para Salgado (2018), los valores son esenciales dentro de la sociedad durante su proceso de cambio, ya que es fundamental pese al paso del tiempo que las costumbres y tradiciones no se pierdan. Específicamente, el actuar idóneo ante las diferentes situaciones de la vida.

Además, se puede evidenciar que los buenos o malos actos se identifican como una guía para los demás individuos. Como Bandura (1977) en su teoría cognitiva, al establecer diferentes modelos de comportamiento, hace mención que los niños observan a los demás para ver e imitar cómo se comportan. Por lo cual, se debe considerar que los niños, niñas, jóvenes y adolescentes están rodeados de muchos modelos a seguir, de quienes pueden imitar tanto las buenas como las malas acciones.

Vygotsky (1978) junto a Bruner (1961) creen fundamental reconocer al docente como el facilitador del desarrollo mental de los estudiantes, quien establece una guía para construir aprendizajes cada vez más complejos. Siendo la información impartida adecuada a sus edades, sumado a la adecuada influencia del ambiente. Por lo que, no solo niños deben contribuir a su formación, sino toda la sociedad y limitar el uso excesivo de las herramientas tecnológicas a tempranas edades.

Lugo (2016) cree que el uso excesivo e inadecuado de dispositivos o herramientas tecnológicas influye de manera directa sobre la capacidad de interacción personal, de expresar sus sentimientos, demostrar empatía. Dificultando así, las relaciones reales generando un círculo vicioso virtual que posteriormente se evidenciará en su actuar, comportamiento, decisiones.

En este sentido, Rivera (2018) recomienda un aprendizaje con valores para así crear conciencia y apoyo en temas referentes a su parte emocional, responsabilidad, consideración, respeto hacia uno mismo y hacía los demás, tolerancia, honestidad. 


\section{CONCLUSIÓN O CONSIDERACIONES FINALES}

Se puede evidenciar que existe una fuerte relación entre las variables estudiadas, los altos niveles obtenidos en la dimensión de Convivencia armónica nos dicen que en referencia a inclusividad y trabajo en equipo, los estudiantes encuestados presentan un gran compromiso. En cambio, se requiere reforzar la dimensión de diversidad. En cuanto a las dimensiones que corresponden a la variable dependiente Desarrollo humano integral, los encuestados aportaron valiosos niveles, donde se puede observar que sus valores éticos, morales y cívicos, espiritualidad son muy altos. En este sentido, aunque los niveles son aceptables se consideró apropiado trabajar y reforzar la dimensión cognitiva y afectiva.

Frente a esta realidad, se requirió estructurar y brindar talleres y charlas, con su respectivo apoyo psicológico. La estrategia implementada ha permitido enriquecer los valores éticos, morales, cívicos, el trabajo en equipo, toma de decisiones autónomas, reconocimiento de sentimientos, empatía, toma de decisiones, reconocimiento $\mathrm{y}$ compresión de su entorno. Estas actividades se han realizado para mejorar el comportamiento de los estudiantes y mejorar la convivencia armónica desde el fortalecimiento de su desarrollo integral.

\section{LISTA DE REFERENCIAS}

Aaros, J. (2003). La ética de Aristóteles y su relación con la ciencia y la técnica. Revista electrónica Diálogos Educativos, Año 3, N6, 2003. ISSN 0718-1310 http://146.83.132.45/articulos/2003/dialogos-e-06-Articulo-Araos-EticadeAristoteles.pdf

Bandura, A. (1977). Teoría del Aprendizaje Social. Madrid, 1982. En Montolla Castillo y Muñoz Iranzo “La iniciativa de habilidades para la vida en el ámbito escolar". Compartim Revista de Formació del Professorat. Valencia, 2009.

Barrios, A. (2016). Concepciones de conflictos interpersonales y desarrollo moral en la educación infantil brasileña. Revista de Psicología (PUCP), 34(2), 261- 291. https://dx.doi.org/10.18800/psico.201602.002

Bruner, J. (1961). El proceso de la educación. Cambridge, Mass: Harvard University Press.

Cf. Platón. La República. https://www.biblioteca.org.ar/libros/8207.pdf 
Cienfuegos, M. y Cienfuegos, A. (2016). Lo cuantitativo y cualitativo en la investigación. Un apoyo a su enseñanza. Ride. Revista Iberoamericana para la Investigación y el Desarrollo Educativo, 7(13), 15-36. http://www.scielo.org.mx/scielo.php?script=sci_arttext\&pid=S200774672016000200015

Díaz, V. y Calzadilla, A. (2016). Artículos científicos, tipos de investigación y productividad científica en las Ciencias de la Salud. Revista Ciencias de la Salud, 14( 1 ): 115-121. http://www.scielo.org.co/pdf/recis/v14n1/v14n1a11.pdf

Kauffman, W. (1975). Existentialism from Dostoevsky to Sartre. A plume book philosophy. ISBN: 978-0-452-00930-1.

Kohlberg, L. (1984). The Psychology of Moral Development: The Nature and Validity of Moral Stages (Essays on Moral Development, Volume 2).

Lugo, A. (2016). Influencia de las redes sociales en el carácter de los niños y adolescentes. Aces educación. http://educacion.editorialaces.com/influencia-delas-redes-sociales-en-elcaracter-de-los-ninos-y-adolescentes/

Martínez, L., Murillo, H., Martínez D., Arciniega, M., Rosales, I., Obregón, L., Núñez, M., Quiñones, A., Pérez, J., Andrade, A., y Méndez, L. (2018). Filosofía, valores, ética, moral e identidad. Universidad Pedagógica de Durango, 2018. ISBN:

978-607-97054-9-7. http://www.redie.mx/librosyrevistas/libros/eticayvalores.pdf

Miño, L. (2018). Los códigos de convivencia escolar: una herramienta para la educación con enfoque de derechos. Revista Tramando. https://www.tramared.com/revista/items/show/36

Murillo, W. (2008). La investigación científica. Ed. Semphis, Madrid

Piaget, J. (1983). El criterio moral en el niño. Editorial Fontanella.

Rivera, D. (2018). Aprendamos con valores. [tesis de Maestría, Universidad Nacional de Educación]. Repositorio Institucional UNAE. http://repositorio.unae.edu.ec/bitstream/56000/640/1/TFM-OE-55.pdf

Salgado, G. (2019). La importancia de la educación en valores en la formación de las nuevas generaciones. Revista Pensamiento libre. ISSN: 2007-5685. https://www.revistapensamientolibre.com/single-post/2019/07/17/laimportanciade-la-educacio-n-en-valores-en-la-formacio-n-de-las-nuevasgeneraciones 
Sampieri, R. (2014). Metodología de la investigación. McGRAW-HILL / INTERAMERICANA EDITORES, S.A. DE C.V. Sexta edición. ISBN: 978-14562-2396-

http://observatorio.epacartagena.gov.co/wpcontent/uploads/2017/08/metodologia -de-la-investigacion-sextaedicion.compressed.pdf

Vygotsky, L. (1931). Teoría de las emociones. Ediciones Akal / 978-84-460-1299-3

Vygotsky, L. (1978). Mind in Society: The Development of Higher Psychological Processes. Cambridge. 